\title{
CORRESPONDENCE
}

\section{Acute exacerbations of COPD: it's the weekend but it can't wait until Monday}

\section{To the Editors:}

In a large nationwide study in Spain, BARBA et al. [1] recently reported that patients admitted for treatment of an acute exacerbation of chronic obstructive pulmonary disease (AECOPD) over a weekend showed poorer in-hospital survival compared to those admitted on weekdays. The data suggest that this effect is independent of confounders such as age, sex, comorbidity and respiratory failure. The authors elaborate on the possibility that limited healthcare availability during the weekends may be an explanation for their findings.

However, we believe that the data of BARBA et al. [1] support an additional explanation. Chronic obstructive pulmonary disease patients with respiratory distress during the weekend may tend to wait until the Monday to contact health services. While the level of symptoms would allow a patient with mild AECOPD to do so without running a significant risk, patients with severe AECOPD ultimately are left no choice but to contact health services during the weekend. Consequently, this shift towards fewer but more severe AECOPD patients presenting during the weekends may contribute to the explanation of the "weekend effect". Indeed, BARBA et al. [1] reported the lowest proportion of admissions at weekends and the highest on Mondays, and the latter had the lowest risk of mortality. Also, patients admitted over the weekends had respiratory failure more often. Although the effect of weekends on mortality remained significant after adjusting for respiratory failure, the lack of data on blood gas analyses, oxygen therapy, medication prescribed and intensive care unit admissions precluded a detailed analysis on the effect of AECOPD severity in this context.

We recommend providing patients with a written action plan [2] with clear instructions and contact details in order to better prepare the patient for the occurrence of an AECOPD, especially outside office hours.

\section{Bram van den Borst ${ }^{*, *}$ and Geertjan Wesseling*,}

*Dept of Respiratory Medicine, Maastricht University Medical Center+, "NUTRIM School for Nutrition, Toxicology and Metabolism, and "CAPHRI School for Public Health and Primary Care, Maastricht, The Netherlands.

Correspondence: G. Wesseling, Maastricht University Medical Centre, P.O. Box 5800, Maastricht, the Netherlands. E-mail: g.wesseling@mumc.nl

Statement of Interest: None declared.

\section{REFERENCES}

1 Barba R, Zapatero A, Losa JE, et al. The impact of weekends on outcome for acute exacerbations of COPD. Eur Respir J 2012; 39: 46-50.

2 Trappenburg JC, Monninkhof EM, Bourbeau J, et al. Effect of an action plan with ongoing support by a case manager on exacerbation-related outcome in patients with COPD: a multicentre randomised controlled trial. Thorax 2011; 66: 977-984.

DOI: $10.1183 / 09031936.00022612$

\section{Could neutrophilic airway inflammation in obese people be more due to obstructive sleep apnoea syndrome than to asthma?}

\section{To the Editors:}

The study by ScOTT et al. [1] investigated the known association between asthma and obesity with the aim of better understanding the mechanism that drives airways inflammation in these conditions. They enrolled obese and nonobese subjects with asthma and obese and nonobese healthy controls, supposing that obese asthmatics could have a different airway inflammation phenotype compared with nonobese asthmatics. They described an increase in sputum neutrophils in obese asthmatics attributed to the innate immune pathways, while the sputum eosinophils were unaffected by obesity. Furthermore, they reported the highest concentrations of plasma interleukin (IL)-6, C-reactive protein and leptin in 
obese asthmatic subjects, which confirms that airway inflammation coexists with systemic inflammation in these subjects. Another important finding of this study was the greater percentage of neutrophils, which was especially observed in obese asthmatic females compared with nonobese subjects.

In this regard, although the excess fat remains a potential cause of systemic and local inflammation in asthma, it is now largely known that obesity is one of the main risk factors for obstructive sleep apnoea syndrome (OSAS), a condition that has a similar increase in neutrophilic airways inflammation in its pathogenesis [2]. In their article, ScOTT et al. [1] found an increase in neutrophilic inflammation mainly in females, a group where the prevalence of OSAS is lower. However, females of perimenopausal age, such as those enrolled by SCOTT et al. [1], are more susceptible to developing OSAS [3].

The authors of this study completely ignored the possibility that the obese subjects enrolled could also have obstructive sleep apnoea and that it could justify the development of the airways inflammation observed.

Several studies have investigated the association between airway inflammation and OSAS, describing an increase in neutrophils in sputum [2], and high concentrations of exhaled nitric oxide, exhaled carbon monoxide, and other inflammatory markers in the exhaled breath condensate (EBC), such as $\mathrm{pH}[4]$, leukotriene $\mathrm{B}_{4}$, tumour necrosis factor- $\alpha$, IL-6 and IL-8, in subjects with OSAS, especially when obese $[4,5]$.

Most of these markers correlated with the severity of OSAS and decreased after continuous positive airway pressure therapy, confirming that they are related more to sleep apnoea than to obesity [2]. Our group further showed an increase in intercellular adhesion molecule-1 in the EBC of OSAS patients, attributing the leukocyte migration and sustainment of local inflammation in their airways to this cell adhesion molecule [5].

Furthermore, in support of their hypotheses, ScOTT et al. [1] showed that body fat may drive airway inflammation in obese asthmatic females, involving an increase in plasmatic leptin in obese asthmatic females. However, as they acknowledge, airway leptin levels might be important to link this obesity to the airway inflammation, but they did not perform these measurements. An increase in leptin in the plasma of obese patients with OSAS compared to obese subjects without OSAS was recently described; this constitutes further proof that the airway inflammation found in obese asthmatics could be due to an overlap in these patients with OSAS rather than to the obesity itself [6]. Although in this study, as in a previous one [7], the percentage of neutrophils was higher than in the control group, they were still in the normal range. These data suggest that the presence of neutrophils is only one aspect of airway inflammation.

Moreover, even if there are not, to our knowledge, any studies that have investigated the relationship between hyperlipidaemia and airway inflammation, we cannot exclude that this could represent another potential factor linking obesity and airway inflammation in asthmatic females.

Another limitation, also recognised by the authors of this study, was that they did not measure the body fat distribution but only considered the body mass index (BMI). As is widely known, obese subjects often present an increase in neck circumference that becomes a high-risk factor for sleep apnoea, and should perhaps always be investigated in obese subjects.

In this regard, the fact that the enrolled population (obese subjects with asthma and also without asthma) was age-, sexand BMI-matched does not mean that they have the same nocturnal characteristics in terms of respiratory disorders or that these variables are simply the consequence of asthma. Indeed, it is also necessary to consider the conformational alterations of facial skeletons and, particularly, the evaluation of fat distribution, neither of which were assessed in the study by ScOTT et al. [1]; it is known that the neck circumference, more than weight, is important in causing nocturnal respiratory alterations [8].

In conclusion, we would underline that, although the neutrophilic asthma reported by ScOTT et al. [1] could be a particular phenotype observed in females with high BMI, as previously suggested both by the European Network for Understanding Mechanisms of Severe Asthma study [7] and Holguin et al. [9], possible clinical consequences of OSAS should also be investigated, especially when the asthma is not well-controlled.

\section{Giovanna Elisiana Carpagnano*, Maria Pia Foschino- Barbaro", Pierluigi Carratu", Donato Lacedonia and Onofrio Resta?}

*Dept of Thoracic Medicine, "Institute of Respiratory Diseases, Dept of Medical and Occupational Sciences, University of Foggia, and Dept of Pulmonary and Critical Care Medicine, University of Bari, Bari, Italy.

Correspondence: G.E. Carpagnano, Universita di Foggia, Via de Nicolo 5, Bari, Italy, Viale del Tamerice 7, Palese, Bari, Italy, Bari, Italy, 70121. E-mail: ge.carpagnano@unifg.it

Statement of Interest: None declared.

\section{REFERENCES}

1 Scott HA, Gibson PG, Garg ML, et al. Airway inflammation is augmented by obesity and fatty acids in asthma. Eur Respir J 2011; 38: 594-602.

2 Carpagnano GE, Lacedonia D, Foschino-Barbaro MP. Non-invasive study of airways inflammation in sleep apnea patients. Sleep Med Rev 2011; 15: 317-326.

3 Kapsimalis F, Kryger MH. Gender and obstructive sleep apnea syndrome, part 2: mechanisms. Sleep 2002; 25: 499-506.

4 Carpagnano GE, Spanevello A, Sabato R, et al. Exhaled $\mathrm{pH}$, exhaled nitric oxide, and induced sputum cellularity in obese patients with obstructive sleep apnea syndrome. Transl Res 2008; 151: 45-50.

5 Carpagnano GE, Spanevello A, Sabato R, et al. Systemic and airway inflammation in sleep apnea and obesity: the role of ICAM-1 and IL-8. Transl Res 2010; 155: 35-43.

6 Antonopoulou S, Loukides S, Papatheodorou G, et al. Airway inflammation in obstructive sleep apnea: is leptin the missing link? Respir Med 2008; 102: 1399-1405. 
7 The ENFUMOSA cross-sectional European multicentre study of the clinical phenotype of chronic severe asthma. Eur Respir J 2003; 22: 470-477.

8 Simpson L, Mukherjee S, Cooper MN, et al. Sex differences in the association of regional fat distribution with the severity of obstructive sleep apnea. Sleep 2010; 33: 467-474.
9 Holguin F, Bleecker ER, Busse WW, et al. Obesity and asthma: an association modified by age of asthma onset. J Allergy Clin Immunol 2011; 127: 1486-1493.

\section{EBUS-TBNA in the differential diagnosis of pulmonary artery sarcoma and thromboembolism}

\section{To the Editors:}

We read with interest the correspondence published by PARK et al. [1] suggesting a potential role for endobronchial ultrasound-guided transbronchial needle aspiration (EBUSTBNA) to differentiate pulmonary artery sarcoma and thromboembolism.

Pulmonary artery sarcoma usually has its origin in the pulmonary arterial trunk and extends towards pulmonary branches, and is indeed sometimes difficult to differentiate from chronic thromboembolic disease. However, we believe that the authors' conclusions suggesting that EBUS-TBNA is safe in this setting are premature. Indeed, such an approach could be associated with significant complications.

Even if pulmonary artery sarcoma may mimic acute or chronic pulmonary thromboembolism, it has been reported $[2,3]$ that a careful analysis including medical history, chest computed tomography (CT), magnetic resonance imaging (MRI), pulmonary angiogram and positron emission tomography CT with ${ }^{18}$ F-2-fluoro-2-deoxy-D-glucose [4,5] may be sufficient to detect patients with pulmonary artery sarcoma. Chest CT classically shows hyperdense lesions, beaded peripheral pulmonary arteries, and contiguously soft tissue-filled pulmonary arteries and extravascular spread [2]. Moreover, MRI may be more specific for pulmonary artery sarcoma, showing enhancement with gadolinium contrast [2].

PARK et al. [1] performed EBUS-TBNA in a patient with acute pulmonary embolism, which was later confirmed by the complete reversibility of endovascular clots after 6 weeks of anticoagulation. Performing EBUS-TBNA in a patient with acute thromboembolism is certainly debatable and hazardous, and it is important to state that a diagnosis of acute thromboembolic disease is based on established imaging techniques, such as ventilation/perfusion lung scan, chest CT and pulmonary angiography [6].

Diagnosis of an endovascular tumour could be made by endovascular catheter biopsy [7]. In addition, as stated by PARK et al. [1], surgery is the treatment of choice in the management of pulmonary artery sarcoma (pneumonectomy, pulmonary artery resection/reconstruction or pulmonary endarterectomy) and chronic thromboembolic pulmonary disease (pulmonary endarterectomy) [6]. It is therefore important to highlight that surgery should be proposed to eligible patients with pulmonary artery sarcoma or chronic thromboembolic pulmonary hypertension, allowing diagnosis confirmation and management.

Finally, a large proportion of patients with proximal pulmonary artery chronic obstruction by sarcoma or thromboembolic material may present with pulmonary hypertension, a condition associated with a high risk of complication following transbronchial needle aspiration. Indeed, it has been clearly demonstrated that proximal obstruction of pulmonary arteries may be associated with hypertrophy of systemic bronchial arteries (fig. 1), increasing the risk of haemorrhage from transbronchial needle aspiration.

To conclude, we believe that physicians should be aware of the potential complications of EBUS-TBNA in patients with pulmonary artery sarcoma or pulmonary thromboembolic disease, and we consider that EBUS-TBNA should not be

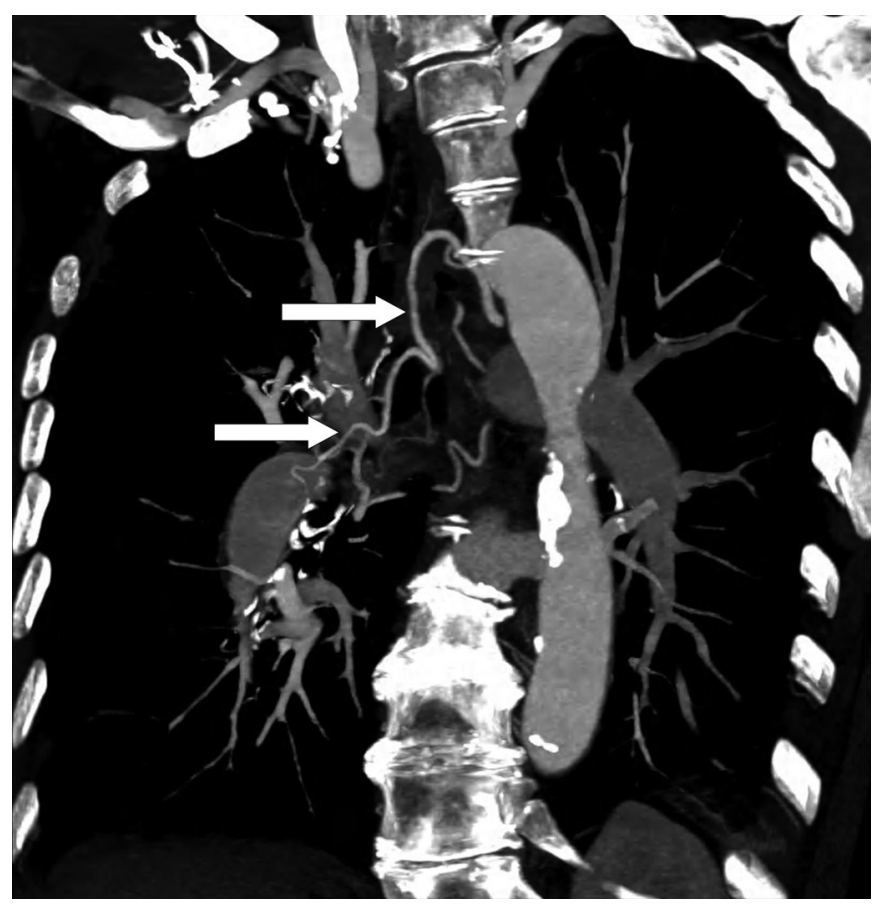

FIGURE 1. Computed tomography angiography with maximum-intensity reconstruction in the coronal plane showing hypertrophy of systemic bronchial arteries (arrows) in a case of proximal chronic thromboembolic pulmonary disease. 